\title{
Okadaic Acid Induces the Rats' Memory Impairment and Tau Protein Hyperphosphorylation And Intervened by Flavonoids from Stem and Leaf of Scutellaria Baicalensis Georgi
}

\author{
Cheng Jianjun and Shang Yazhen* \\ Institute of Traditional Chinese Medicine, Chengde Medical College, China
}

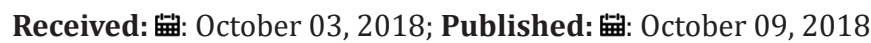

${ }^{*}$ Corresponding author: Shang Yazhen, Institute of Traditional Chinese Medicine, Chengde Medical College/Hebei Province Key Research Office of Traditional Chinese Medicine Against Dementia/Hebei Province Key Laboratory of Traditional Chinese Medicine Research and Development, Chengde, Hebei 067000, PR China

Abbreviations: AD: ALZHEIMER'S DISEASE; NFTs: Neurofibrillary Tangles; P-Tau: Phosphorylated Tau; SP: Senile Plaques; PHF: Paired Helical Filament; CDK5: Cyclin-Dependent Kinase 5; Tyr: phosphorylation at tyrosine; Ser: serine; PKA: protein kinase; GSK3 $\beta$ : Glycogen Synthase Kinase 3ß; OA: Okadaic Acid

\section{Introduction}

Alzheimer's disease (AD) is a neurodegenerative disease characterized by progressive cognitive impairment. The neuropathology of $\mathrm{AD}$ is represented by widespread deposits of neurofibrillary tangles (NFTs) formed by phosphorylated tau (p-tau) protein and of senile plaques (SP) containing amyloid $\beta(A \beta)$ protein. A series of epidemiological statistical results and the experimental study showed that solely SP cannot result in $\mathrm{AD}$ clinical dementia symptoms, while the formation and the number of NFTs is directly related to the occurrence of dementia. NTFs formation is due to excessive phosphorylated tau protein occurred after the formation of the structure of the double helix shape. Actually, tau, a microtubule-binding protein, primarily promotes microtubule stability and contains 2-3 phosphate groups per molecule in the normal brain. However, in the AD brain, tau protein hyperphosphorylated, containing 5-9 phosphate groups per molecule, has a decreased ability to combine with microtubules and dimerize.

Stable tau dimers form tau oligomers, which continue aggregating to form subunits of filaments called protomers. Two protomers wound around each other form a paired helical filament (PHF), and excessive PHF assembly leads to NFTs and eventually $\mathrm{AD}$ [1]. An imbalance between tau phosphorylation and dephosphorylation is critical to AD tauopathy. Several protein kinases, including cyclin-dependent kinase 5 (CDK5), cyclic AMPdependent protein kinase (PKA) and glycogen synthase kinase
$3 \beta$ (GSK3 $\beta$ ) have been confirmed to phosphorylate tau protein at different sites, such as at serine (Ser) 199, Ser202, Ser262, Ser396, Ser214, Ser404, Thr231 and other sites of tau protein. These forms of p-tau protein are found in the $\mathrm{AD}$ brain. In addition, the phosphorylation of protein kinases at different sites can alter their kinase activity. For instance, GSK3 $\beta$ activity is inhibited by phosphorylation at serine (Ser) 9, and its activity is enhanced by phosphorylation at tyrosine (Tyr) 216. Hyperphosphorylated tau protein also results from the reduced activity of protein phosphatase (PP2A, PP2B, PP2C, PP1) that indicates a decisive role of $\mathrm{PP}$ in tangle formation in $\mathrm{AD}$. Then any strategy that can maintain hyperphosorylation of tau protein can be used to establish an ADlike animal model for studying pathophysiology process and drug screen with AD [2].

Okadaic acid (OA), a polyether toxin isolated from marine microalgae, is a selective inhibitor of PP and is neurotoxic. An AD-like animal model was developed by intracerebroventricular injection of $\mathrm{OA}$, which resulted in learning and memory deficits, tau hyperphosphorylation, and NFT accumulation in the brain. This ADlike animal model can be regarded as an accurate simulation of the pathophysiology of AD in vivo [3]. SSF, a flavonoid isolated from the stems and leaves of Scutellaria baicalonsis Georgi, has demonstrated beneficial effects towards cognitive improvement in animals with cognitive deficits induced by $\mathrm{AlCl} 3, \mathrm{D}$-galactose and aggregated $\mathrm{A} \beta$ [4-6]. The present study established an AD-like animal model via 
the injection of $\mathrm{OA}$ into the right lateral cerebral ventricle of rats. We investigated the effects of SSF on memory impairment, neuron injury, NFT deposition, tau protein phosphorylation at Ser199, Ser202, Ser214, Ser262, Ser396, Ser404 and Thr231 in the brain and examined the effects of SSF on the regulatory mechanisms of CDK5, PKA, GSK3 $\beta$ and PP.

The results of the present study showed that the intracerebroventricular injection of $\mathrm{OA}$ to rats can result in the deterioration in rats' memory acquisition, memory retention and memory re-learning; damages in neuron structure; the increases of PHF, p-tau(Ser199), p-tau(Ser202), p-tau(Ser214), p-tau(Ser262), p-tau(Ser396), p-tau(Ser404), p-tau(Thr231) and pTyr216-GSK3 $\beta$ protein expression; as well as the decreases of PKA and pSer9GSK3 $\beta$ protein expression. The protein expression of CDK5 was not significantly changed by $\mathrm{OA}$ treated. Concerning to the PP, the intracerebroventricular injection of $\mathrm{OA}$ to rats can markedly decrease protein expression of PP2A-C $\alpha$, PP2A-C $\beta$, PP2CB and PP1 of the brain. However, SSF treated the rats for $36 \mathrm{~d}$ to reverse OA-resulted in memory impairment, neuron injury, the increased PHF, p-tau(Ser199), p-tau(Ser202), p-tau(Ser214), p-tau(Ser262), p-tau(Ser396), p-tau(Ser404) p-tau(Thr231) and pTyr216-GSK3 $\beta$ protein expression, the decreased PKA, pSer9-GSK3 $\beta$, PP2A-C $\alpha$, PP2A-C $\beta$, PP2CB and PP1 protein expression [7-9].

The current studies demonstrated that SSF is improved memory deficits and neuroprotective against OA primary from lowering the hyperphosphorylation of tau protein and regulative balance of protein kinases and protein phosphatase activites and then inhibiting PHF increase. These findings, coupled with our previous studies, indicate that the action of SSF is based on multiple targets, which may be predicted beneficial effects for the treatment of AD.

\section{Acknowledgement}

The authors wish to thank Hebei Provincial Education Department (No. ZD20131022) and Hebei provincial Hundred

\section{ISSN: 2574-1241}

DOI: $10.26717 /$ BJSTR.2018.09.001842

Shang Yazhen. Biomed J Sci \& Tech Res

cC $(9)$ This work is licensed under Creative

Submission Link: https://biomedres.us/submit-manuscript.php outstanding Innovated Talent's program(First Batch) of China for financial support.

\section{References}

1. Takeda S (2018) Progression of Alzheimer's disease, tau propagation, and its modifiable risk factors. Neurosci Res.

2. Ma RH, Zhang Y, Hong XY, Wang JZ, Liu GP, et al. (2017) Role of microtubule-associated protein tau phosphorylation in Alzheimer's disease. J Huazhong Univ Sci Technolog Med Sci 37(3): 307-312.

3. Zhang SF, Dong YC, Zhang XF, Wu XG, Guan LH, et al. (2015) Flavonoids from Scutellaria attenuate okadaic acid-induced neuronal damage in rats. Brain Inj 29(11): 1376-82.

4. Shang YZ, Miao H, Cheng JJ, Li ST, Wang BY (2005) Effects of SSF on memory deficits in aluminum toxic mice. Chin Pharmacol Bull 21(3): 361-365.

5. Shang YZ, Gong MY, Zhou XX, Li ST, Wang BY (2001) Improving effects of SSF on memory deficits and pathological changes of neural and immunological systems in senescent mice. Acta Pharmacol Sin 22(12): 1078-1083.

6. Wu XG, Wang SS, Miao H, Jian J Cheng, Shu F Zhang, et al. (2016) Scutellaria barbata flavonoids alleviate memory deficits and neuronal injuries induced by composited $A \beta$ in rats. Behav Brain Funct 8 12(1): 33.

7. Cheng JJ, Zhong HX, Guo K, YZ Shang (2016) Flavonoid extract from Scutellaria stem and leaf attenuates composited $A \beta$ - induced memory impairment and apoptosis in rats. Chin J New Drugs 25(22): 2627-2636.

8. Wang XQ, Gao Yang, Dong YC, et al. (2018) Flavonoids from Scutellaria baicalensis Stems and Leaves Inhibit PHF abnormalities and regulatory mechanism of protein Phosphatase in rats induced by okadaic Acid. Chin J Pathophys 34(01): 94-100.

9. Gao Y, Wang XQ Cao QY Flavonoids from stem and leaf of Scutellaria baicalonsis Georgi inhibit the phosphorylation on multi-sites of tau protein induced by okadaic acid and the regulative mechanism of protein kinases in rats. In preparation.

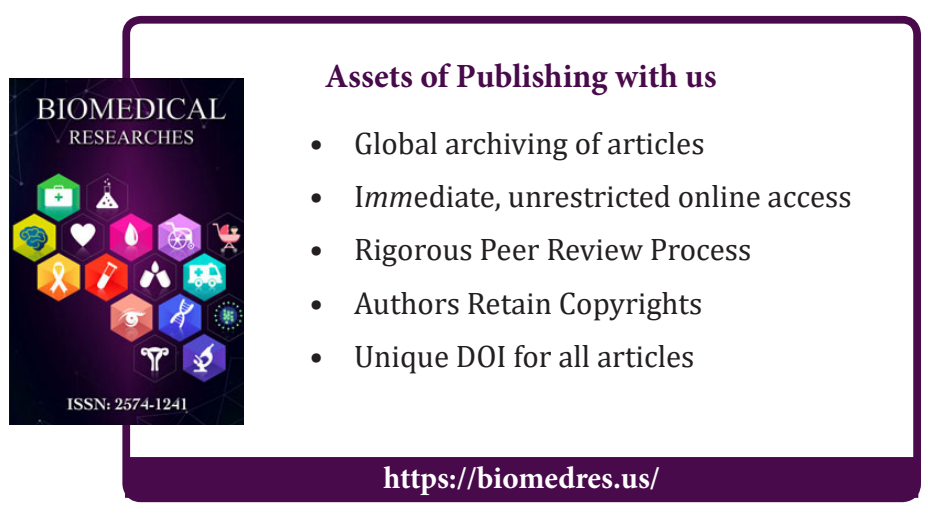

Cite this article: Cheng Jianjun, Shang Yazhen. Okadaic Acid Induces the Rats' Memory Impairment and Tau Protein Hyperphosphorylation And Intervened by Flavonoids from Stem and Leaf of Scutellaria Baicalensis Georgi. Biomed J Sci\&Tech Res 9(4)-2018. BJSTR. MS.ID.001842. DOI: 10.26717 / BJSTR.2018.09.001842. 